\title{
Extraspinal Incidental Findings at Lumbar Spine Magnetic Resonance Imaging in Two Hospitals: Prevalence and Clinical Importance
}

\author{
Odile Fernande Zeh1,2,3*, Emilienne Guegang Goujou, 1,4, Armel Philippe Awana1,5,6, \\ Julienne Onguene Medza',2, Joshua Tambe', Claude Sandra Raïssa Abomo Ngodo1, \\ Maxwell Goudjou Sandjong1, Joseph Gonsu Fotsin ${ }^{1,2}$
}

\author{
${ }^{1}$ Faculty of Medicine and Biomedical Sciences, University of Yaounde I, Yaounde, Cameroon \\ ${ }^{2}$ Yaounde Gynaecology Obstetrics and Pediatrics Hospital, Yaounde, Cameroon \\ ${ }^{3}$ Jordan Medical Center, Yaounde, Cameroon \\ ${ }^{4}$ Yaounde General Hospital, Yaounde, Cameroon \\ ${ }^{5}$ Yaounde Jamot Hospital, Yaounde, Cameroon \\ ${ }^{6}$ Jacques Monod Hospital Le Havre, Montivilliers, France \\ Email: *of_zeh@yahoo.fr
}

How to cite this paper: Zeh, O.F., Guegang Goujou, E., Awana, A.P., Onguene Medza, J., Tambe, J., Abomo Ngodo, C.S.R., Goudjou Sandjong, M. and Gonsu Fotsin, J. (2017) Extraspinal Incidental Findings at Lumbar Spine Magnetic Resonance Imaging in Two Hospitals: Prevalence and Clinical Importance. Open Journal of Radiology, 7, 241-248.

https://doi.org/10.4236/ojrad.2017.74026

Received: October 9, 2017

Accepted: December 1, 2017

Published: December 4, 2017

Copyright ( 92017 by authors and Scientific Research Publishing Inc. This work is licensed under the Creative Commons Attribution International License (CC BY 4.0).

http://creativecommons.org/licenses/by/4.0/

cc) (7) Open Access

\begin{abstract}
Objective: To assess the importance of incidental extraspinal findings on Magnetic Resonance Imaging of the lumbar spine in two hospital facilities. Materials and Methods: It was a descriptive and retrospective study from November 2015 to March 2016. The records of patients who had done a Magnetic Resonance Imaging (MRI) scan of the lumbar spine were re-read in search of incidental findings. The incidental findings found were classified using Colonography Reporting and Data System(C-RADS) classification of extracolonic lesions to assess clinical significance. The prevalence of incidental findings was calculated for each facility, as well as the distribution according to age, the organs involved and the clinical importance. A non-detection rate was calculated by confronting the findings of the study with the original reports. Results: The prevalence of incidental findings was respectively 33\% (19 out of 36) in Jordan Medical Center (JMC) in Yaounde and 27.74\% (106 out of 292) in Jacques Monod Hospital. The extraspinal incidental findings were classified mainly as extracolonic 2 (E2): $58 \%$ in each facility. The percentage detection of incidental findings was 5\% at JMS and 1.7\% at Jacques Monod Hospital. Conclusion: Extraspinal incidental findings are frequent in both hospitals. However, the rate of detection remains very low.
\end{abstract}

\section{Keywords}

Incidental Findings, Magnetic Resonance Imaging, Lumbar Spine, 
Extraspinal Lesions

\section{Introduction}

Incidental findings are lesions found outside the region of interest having no link with the request for imaging [1]. Magnetic Resonance Imaging (MRI) of the lumbar spine with the usual protocol (sagittal T2- and T1-weighted slices, axial T2-weighted slices) can prove to be useful in the search of incidental extraspinal lesions.

Several studies have revealed extraspinal incidental findings (IF) after an MRI of the lumbar spine. Quattrochi et al. [1] in 2013 showed that 17.6\% of IF found after a review of MRI scans were clinically important. To classify these IF, he used the extracolonic lesion classification system C-RADS. In addition, other studies have focused their work on the percentage of detection of these lesions and report on the accounts rendered [2] [3].

In Cameroon, MRI equipment is still rare among hospital facilities. Nevertheless, there is a gradual increase in equipment procurement by health facilities compared to France where the equipment is more widespread.

The detection of IF poses a problem of maintenance, because some deserve a considerable monitoring. The purpose of this study is to assess the percentage and the clinical significance of the extraspinal lesions by reviewing lumbar spine MRI scans in two hospitals in Cameroon and in France.

\section{Materials and Methods}

\subsection{Type of Study}

A cross-sectional descriptive study was carried out by reviewing the records of patients who performed an MRI of the lumbar spine from November 2015 to March 2016 in two hospitals in Yaoundé and Le Havre using as equipment a HITACHI APERTO LUCENT 0.5 T and SIEMENS AERAXJ 1.5 T respectively.

The records of patients with repeated scans or incomplete files were excluded from our study.

\subsection{MRI}

MRI scanning protocols were as follows.

\subsubsection{Jordan Medical Center (Cameroon)}

The scans were performed using a Hitachi Aperto Lucent 0.5 Tesla. The sequences were as follows: sagittal T2-weighting (slice thickness: $4 \mathrm{~mm}$; size of the matrix 512), sagittal T1-weighting (slice thickness: $4 \mathrm{~mm}$; size of the matrix 512), axial T2-weighting (slice thickness: $4 \mathrm{~mm}$; size of the matrix 512). Two sequences could have been added, depending on the radiologist on seat: coronal plane T2-weighting (slice thickness: $4.5 \mathrm{~mm}$; size of the matrix 512) and coronal plane 
STIR (slice thickness: $4.5 \mathrm{~mm}$; size of the matrix 320).

\subsubsection{Jacques Monod Hospital of Havre (France)}

The examinations were made on a Siemens AERAXJ of 1.5 Tesla. The protocol was done according to the following sequences: Sagittal T2-weighting (slice thickness: $4 \mathrm{~mm}$; size of the matrix 512), Sagittal T1-weighting (slice thickness: 4 $\mathrm{mm}$; size of the matrix 384), Sagittal STIR (slice thickness: $4 \mathrm{~mm}$; size of the matrix 384), Axial T2-weighting (slice thickness: $4 \mathrm{~mm}$; size of the matrix 384). Two sequences could be added as a function of the Radiologist: coronal plane T2-weighted images (thickness of the slices: $4.5 \mathrm{~mm}$; size of the matrix 512) and coronal plane STIR images (thickness of the slices: $4.5 \mathrm{~mm}$; size of the matrix $320)$.

\subsection{Analysis of the Data}

Records of patients who had an MRI of the lumbar spine done during the study period were re-read. The extraspinal IF found were reported by organ and by system.

Subsequently, these lesions have been classified according to clinical significance using C RADS system (CT Colonography Reporting and Data System) for extracolic lesions. Zalis et al. [2] published a work on "CT Colonography Reporting and Data System". This was based on three points:

- Description of polyps and masses: Morphology, size, location and structure

- Classification of colic lesions (C): C0 (limited review for the study of colon cancer), $\mathrm{C} 1$ (absence of colonic lesion which increases the risk of developing a cancer), C2 (indeterminate polyp), C3 (possible adenoma) and C4 (probably malignant colon mass)

- Classification of extra colic lesions (E): E0 (study is limited), E1 (normal study or anatomical variant), E2 (no clinical importance), E3 (probably without importance with incomplete characterization) and E4 (potentially important lesions).

Finally we sought to find out if these extraspinal IF were noted in the initial records to determine the detection rate.

\section{Results}

The mean age was respectively of 46 years with variances 14 and 74 years at JMC in Yaounde and 52 years with deviations within 18 to 91 years at Jacques Monod Hospital (Le Havre).

IF were found in $33.3 \%$ of 36 patients with a prevalence among women of $45.45 \%$ and $28 \%$ for men. Table 1 presents the prevalence of IF according to sex and the average age per hospital.

The most represented ages were between 40 - 60 years $(47 \%)$ at JMC in Yaounde and more than 60 years $(35.11 \%)$ at Jacques Monod Hospital (Figure 1).

At the Jacques Monod hospital, there are $27.74 \%$ of incidences in Le Havre 
Table 1. Prevalence of IF and average age by sex.

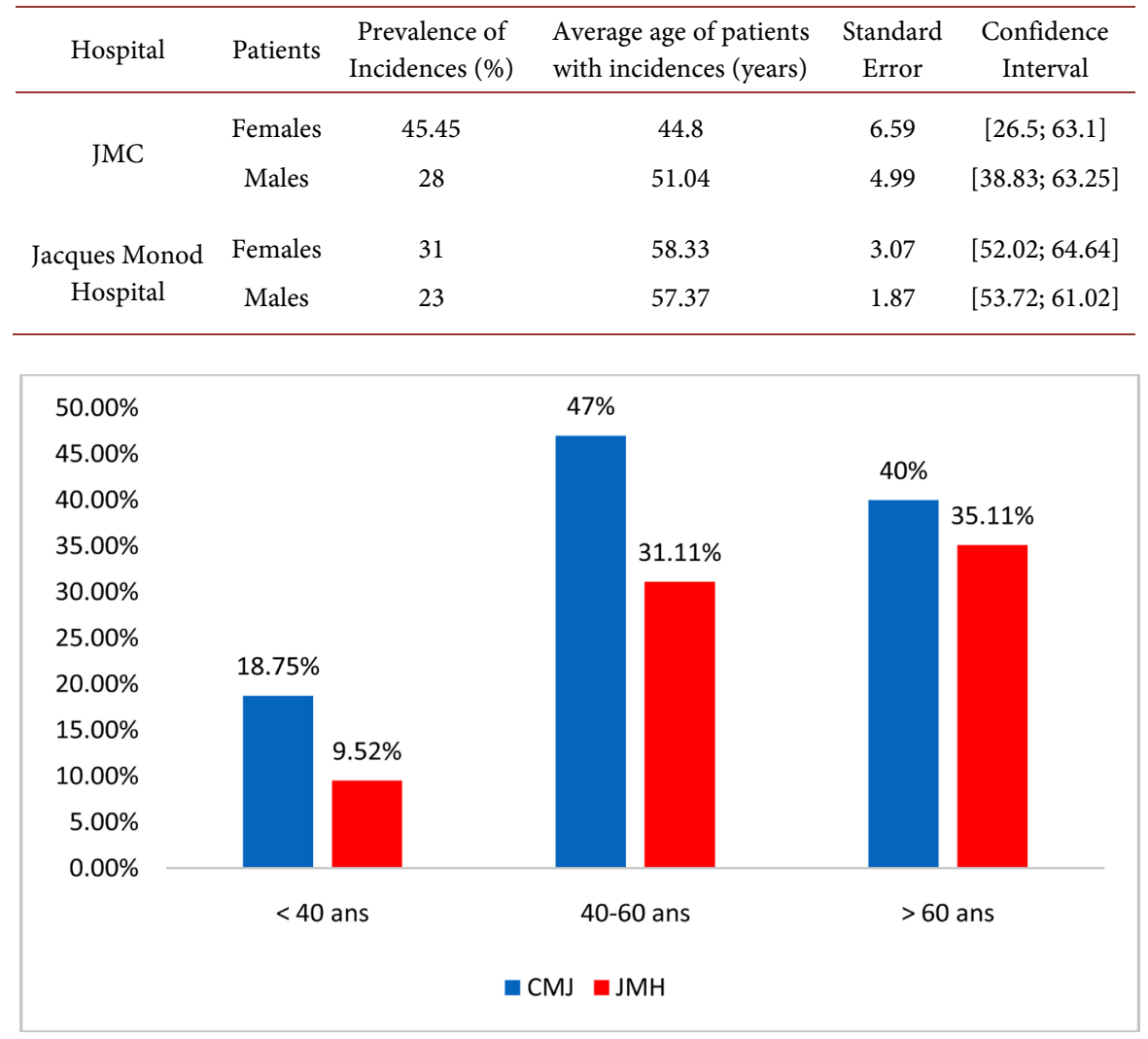

Figure 1. Distribution of IF according to age.

(106 incidences for 81 patients) with a prevalence of $31 \%$ among women and $23 \%$ for men.

Table 2 and Table 3 represented the results of IF with respect to their prevalence according to the C-RADS model.

The most frequent lesions are classified in C-RADS E2, 58\% in Yaounde and $58 \%$ in Le Havre. The small renal cyst was the most represented pathology with 47.2\% in Yaounde and 38.6\% in Le Havre.

Of the 19 IF identified in Yaounde, 3 were classified C-RADS E3: aortic ectasis, aortic thrombus and aortic prosthesis.

No C-RADS E4 was found in Yaounde while a patient in Le Havre presented with an aortic aneurysm.

After record reviewing, the detection percentage was 5\% at JMC in Yaounde (5 out of 19) and 1.7\% at Jacques Monod hospital of Le Havre (1 out of 106).

At the Jacques Monod Hospital, a renal lesion with atypical signal was the most worrying C RADS E3 lesion (Figure 3).

Concerning the lesions C RADS E4, only one lesion was found in Le Havre. It was an aortic aneurysm (Figure 4). This data is closer to that of the study conducted by Quattrochi (0.8\%) [1]. It deviates from the data of Gouliamos which was 3\% [3]. This difference may be due to the use of the saturation band which limits the exploration of the aorta in full. 
Table 2. Classification of IF according to the organs and the clinical significance (JMC).

\begin{tabular}{cccc}
\hline Organs & Incidences & Clinical significance & Percentage \\
\hline \multirow{3}{*}{ Kidney } & Small renal cyst & E2 & $47.3 \%$ \\
& Large renal cyst & E3 & $10.53 \%$ \\
Spleen & Renal pelvis & E2 & $5.26 \%$ \\
& Splenomegaly & E3 & $21.05 \%$ \\
Vascular system & Aortic ectasis & E3 & $5.26 \%$ \\
& Aortic prosthesis & E2 & $5.26 \%$ \\
Total & Aortic thrombus & E3 & $5.26 \%$ \\
\hline
\end{tabular}

Table 3. Classification of IF following the organs and the clinical significance (JMH).

\begin{tabular}{llccc}
\hline \multirow{2}{*}{ Organs } & \multicolumn{1}{c}{ Incidences } & $\begin{array}{c}\text { Clinical } \\
\text { Importance }\end{array}$ & $\begin{array}{c}\text { Number of } \\
\text { Apparitions }\end{array}$ & $\begin{array}{c}\text { Percentage of } \\
\text { Apparition }\end{array}$ \\
\hline \multirow{3}{*}{ Kidney } & - Small renal cyst & E2 & 41 & $38.68 \%$ \\
& - Bilateral renal atrophy & E3 & 3 & $2.83 \%$ \\
& - Large simple renal cyst & E3 & 3 & $2.83 \%$ \\
& - Complex renal cyst & E3 & 2 & $1.89 \%$ \\
& - Absent kidney & E2 & 2 & $1.89 \%$ \\
& - Small myoma & & & $5.66 \%$ \\
& - Naboth's cyst & E2 & 6 & $2.83 \%$ \\
& - Polymyomatous uterus & E3 & 3 & $2.83 \%$ \\
& - Large myoma & E3 & 3 & $0.94 \%$ \\
\hline
\end{tabular}

In Le Havre, the gynecological lesions accounted for the second pathological entity. This prevalence is similar to a study carried out in Poland [4] with 16.7\% of gynecological lesions after a review of 227 MRI for the study of the lumbar spine [5]. Myomas were also found (Figure 5).

After the reviewing of the reports the percentages of detection of IF were very low and were similar to those by Tuncel in 2015 with 7\% [5] and Quattrochi 11\% [1]. European of Radiology could allow an increase of this percentage of detection [6].

\section{Discussion}

\subsection{Prevalence of Incidences}

The prevalence of extraspinal IF of our study seemed to be closer to that found by Seeman et al in 2015 who had found 22\% [3]. Quattrochi et al during a cohort study in Italy had found $68.6 \%$ of IF [1]. This difference can be explained by the fields of exposure wider than possessed the MRI used by Quattrochi et al. Dilli et al in a retrospective study in Turkey note that incidental pathological findings were detected with a percentage of $14.2 \%$ on lumbar spinal MRI [7], due to the 
large sample in their study.

We did not find any gynecological lesion in Yaounde because the field of exposure was not broad enough to analyze this region.

\subsection{Clinical Significance of Incidences}

The extraspinal IF found had clinically variable significance.

During our study, most of the lesions were classified C RADS E2. This trend was stackable to the high prevalence of renal cysts in the two institutions. These data join the work recovered from Quattrochi and Seeman, which demonstrated that the kidney was the organ bearing the most incidences $(70 \%$ and $35 \%$, respectively) [1] [3]. We believe that this high prevalence of renal lesions could be explained by its proximity with the spine, allowing a good analysis especially on the coronal Slices.

At JMC, aortic ectasis with thrombus was found in a patient of 77 years (Figure 2).

At the Jacques Monod Hospital, a renal lesion with a typical signal was the most worrying C RADS E3 lesion (Figure 3). Concerning the lesions C RADS E4, one lesion was found in Le Havre. It was an aortic aneurysm (Figure 4).

This data is closer to that of the study conducted by Quattrochi (0.8\%) [1]. It moves away from the data of Gouliamos which was 3\% [4]. This difference may

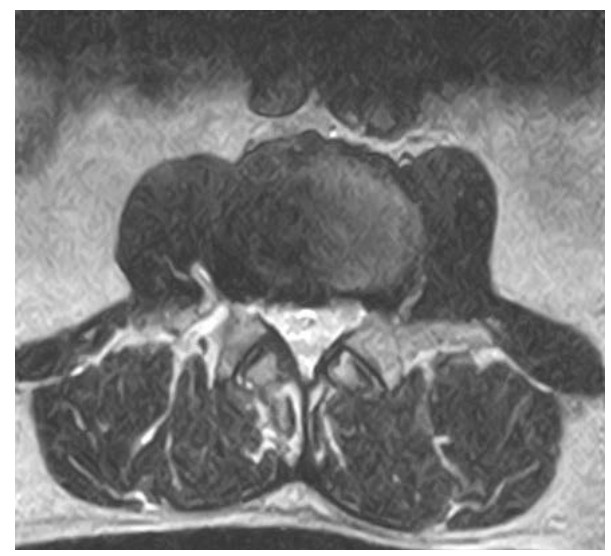

Figure 2. 77-year old male. Axial T2-weighted image passing through the aorta beneath the renal arteries which shows ectasia.

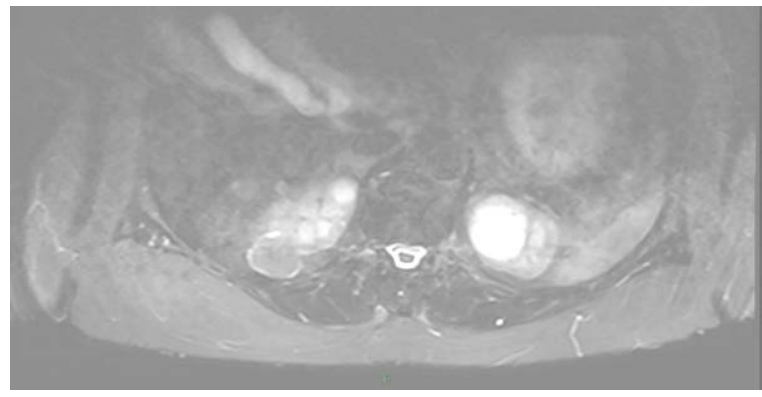

Figure 3. Woman of 62 years. Axial slice in STIR weighting passing through the upper pole of the kidneys shows a right cystic formation that may require complementary imaging. A simple left fibrosis is to be noted. 


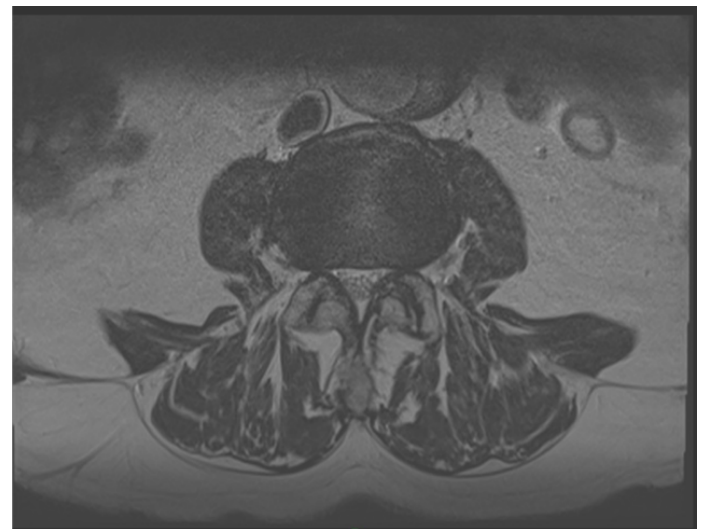

Figure 4. 54-year-old man. Axial T2 weighted slice passing beneath the renal arteries, showing an aortic aneurysm with intraluminal telescopic material that can correspond to a thrombus.

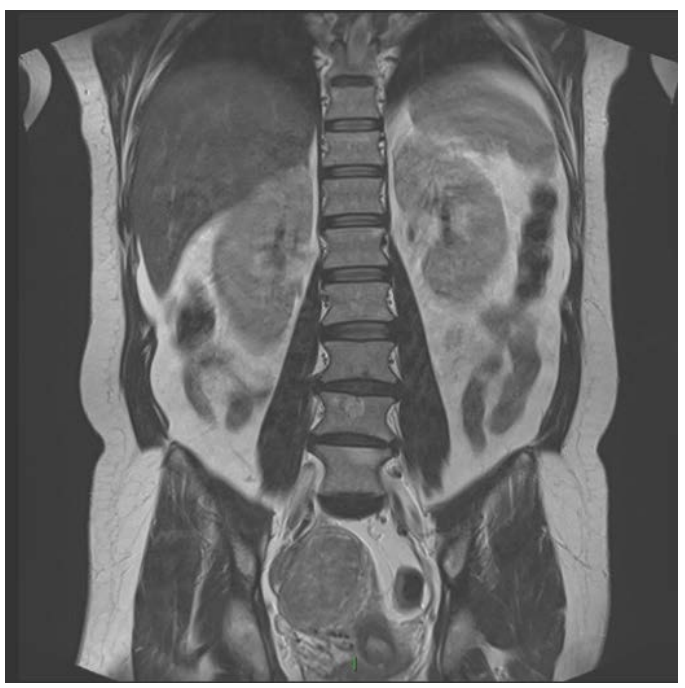

Figure 5. Woman of 46 years. Coronal T2 weighted slice through the psoas muscles showing a large uterine myoma.

be due to the use of the band of saturation which limits the exploration of the aorta in full.

In Le Havre, the gynecological lesions accounted for the second pathological entity. This prevalence is similar to a study carried out in Poland [5] with $16.7 \%$ of gynecological lesions after a reviewing of 227 MRI for the study of the lumbar spine [5]. Myomas had also been found (Figure 5).

\subsection{Rate of Detection}

After the reviewing of the reports, percentage detections of IF were very low and were similar to those by Tuncel in 2015 with 7\% [5] and Quattrochi 11\% [1]. These low percentages of detection could be explained by the fact that the radiologists were focused on the region of interest and a lack of extraspinal IF. They could also have decided to evade these lesions considered mild and not record on the reports. This attitude could be understood, the majority of the lesions 
being classified C RADS E2.

It is necessary to mention some lesions including C RADS E3 and E4 for a follow-up or adequate maintainance. The model of the structuring of the accounts of the European Society of Radiology could allow an increase of this percentage of detection [6].

At JMC, an aortic ectasis with thrombus was found in a patient of 77 years (Figure 2).

\section{Conclusion}

At the end of our study, we concluded that the IF was frequent in the two hospital facilities. Nevertheless, their rate of detection, however, remains very low. But on MRI examination of lumbar spine, paying attention to incidentally detected pathological extraspinal findings is very important due to the fact that they can alter the treatment of the patient.

\section{Conflicts of Interest}

The authors declare they have no conflict of interest.

\section{References}

[1] Quattrochi, C. and Giona, A. (2013) Extra Spinal Incidental Findings at Lumbar Spine MRI in the General Population: A Large Cohort Study. Insights Imaging, 4, 301-308. https://doi.org/10.1007/s13244-013-0234-z

[2] Seeman, H. and Jacob, E. (2015) Incidental Extraspinal Findings at Lumbar Spine Magnetic Resonance Imaging. Spine, 40, 1436-1443.

https://doi.org/10.1097/BRS.0000000000001024

[3] Gouliamos, A.D., Tsiganis, T. and Dimakakos, P. (2004) Screening for Abdominal Aortic Aneurysms during Routine Lumbar CT Scan: Modification of the Technical Standard. Clinical Imaging, 28, 353-355. https://doi.org/10.1016/S0899-7071(03)00316-4

[4] Bekiensińka, M., Wagiel, K. and Adamczyk, Mr. (2004) Incidental Gynecological Findings on Lumbar Spine MRI. Polish Journal of Radiology, 69, 66-70.

[5] Tuncel, S., Cagli, B. and Tekatas, A. (2015) Extraspinal Incidental Findings on MRI Routine of Lumbar Spine: Prevalence and Reporting Rates in 1278 Patients. Korean Journal of Radiology, 16, 866-873. https://doi.org/10.3348/kjr.2015.16.4.866

[6] European Society of Radiology (2011) Good Practice for Radiological Reporting. Guidelines from the European Society of Radiology (ESR). Insights Imaging, 2, 93-96. https://doi.org/10.1007/s13244-011-0066-7

[7] Dilli, A., Ayaz, U., Turanli, S., Saltas, H., Karabacak, O., Damar, C., et al. (2014) Incidental Extraspinal Findings on MRI of Intervertebral Discs. Archives of Medical Science, 10, 757-763. https://doi.org/10.5114/aoms.2014.44868 